\title{
A Novel Approach to informing the Future Land Force: Incorporating Design Thinking and Concept, Development and Exploration Methods
}

\author{
C.J. Manning ${ }^{\mathrm{a}}$, A. Stephens ${ }^{\mathrm{a}}$ and M.K. Richmond ${ }^{\mathrm{a}}$ \\ a Joint and Operations Analysis Division, Defence Science and Technology Group \\ Email: christopher.manning@dst.defence.gov.au
}

\begin{abstract}
The future is complex and uncertain, with emerging technologies that present significant potential advantages as well as threats. In the face of such rapid change, the land force must be able to continue to operate now and be able to adapt and innovate to achieve success in the future. So how can the ADF evolve and both incorporate incremental improvements and embrace radical step change and divestment in order to invest in new concepts that exploit emerging technology?

This paper describes DST Group and Army Headquarters' collaboration to design an aspirational Battle Group (BG) that could achieve success within the 2035 Australian Primary Operating Environment (2035 POE). Key aspects of the 2035 POE are pervasive unmanned aerial systems and sensors more generally, increased lethality and availability of precision munitions, increased capabilities to counter fires, increased availability of electronic warfare effects, and advances in networking leading to highly collaborative effects. Additionally it was assumed that autonomy and AI advances would enable reduction in cognitive burden alleviating the need for tele-operation or remote control, enabling greater numbers of Robotic and Autonomous Systems (RAS) resident within the 2035 BG without reducing the number of combat personnel. Significantly, potential adversary use of these same capabilities would not be constrained by the same legal and ethical constraints as the ADF.

For DST Group, this represents a change in focus to designing concepts and developing force options in addition to assessing them to inform capability decisions. As a result we developed the Design, Concept Development and Exploration (DCDE) approach which took aspects of the NATO Concept Development and Experimentation process, US Army Campaign of Learning approach and UK Disruptive Technology Assessment Games and combined them with Design Thinking methods. A key difference of the DCDE approach is the use of wargaming as an immersive method for concept exploration to exploit emerging technology. The DCDE approach included four key phases; align, design, analyse and refine, which were iterated with the refine phase running concurrently through the entire campaign. This is similar to the Design Thinking philosophy of understand, define, ideate, prototype and test. Flexibility and agility was built into the DCDE approach through the use of multiple methods for analysis with each activity designed to incorporate and respond to findings of preceding activities.
\end{abstract}

The prototype 2035 BG designed using the DCDE approach, balanced novel concepts with current constraints of legacy systems and structures. The 2035 BG gained significant advantage beyond the current 2028 Integrated Investment Program realised (funded) force through use of numerous RAS enabled systems to provide enhanced ISR, EW and fires down to the lowest possible level. 2035 BG concepts included: use of enhanced networking and agile $\mathrm{C} 2$ to achieve both survivability and lethality effects at the lowest levels, use of integrated Counter Rocket Artillery and Missile Systems and Counter Unmanned Air Systems; offensive and defensive EW including deception and decoy tactics. The emerging technology areas and corresponding concepts identified for further investigation are consistent with findings from other studies but have also been contextualised for the 2035 POE.

The DCDE approach represents a novel approach to design a force combining iterative Design Thinking methods and wargaming to provide immersion in context for exploration before detailed testing and assessment. Key benefits of the DCDE approach are flexibility, efficiency, responsiveness and a closer alignment with decision maker needs. The DCDE approach applies a range of methods, models and tools to build an evidence base for modernisation and investment decisions.

Keywords: Force design, Concept development, Wargaming, Red teaming, Military experimentation 


\section{INTRODUCITON}

The Australian Foreign Policy White Paper notes that 'Change, unprecedented in its scale and pace, is the tenor of our times' (Australian Government 2017). GEN Angus Campbell, Chief of the Australian Defence Force, said 'adaptation requires more than just smart people. It requires a permissive, supportive, culture of excellence and innovation. Innovation sees adaptation occur through embrace of radical step change... [and] evolution sees gradual, incremental changes, building on improvements from what has gone before or currently exists' (Campbell 2017). Both adaptation and evolution are key considerations for future force design because the ADF must be successful now and in the future.

The challenges of future force design are compounded by the need to explore concepts based on technologies that have yet to be fielded in order to inform decisions beyond the current Integrated Investment Program (IIP). This paper reports on the 2018 Analysis Campaign (2018 AC), which focused on the design and assessment of a Battle Group (BG) for the 2035 Australian Primary Operating Environment (2035 OE), which represents a specific example of the challenge of future force design for Army at the BG level.

\subsection{The Challenge}

Challenges for Army and DST Group in the conduct of the 2018 AC included the need to develop new concepts and structures within 12 months, balancing a desire for exploration of new capabilities whilst respecting the constraints of the current force, leverage existing joint and coalition experimentation and military exercises and providing detailed quantitative evidence using simulation.

The development of military concepts has often proved challenging; as noted by Greenwood and Greer 2018 "Developing, validating, and ultimately implementing warfighting concepts is extremely challenging for any military service.". Schmitt 2002 suggests that 'military concepts tend to form iteratively and incrementally over time ... It is not an orderly, sequential process'. Smith 2018 challenges existing approaches to force design saying that comprehensive future operating concepts can suppress innovation. Further, Smith 2018 advocates for a contest of ideas with professional debate of limited scope explorative concepts, aggregating resultant innovations and seeking opportunities. As Sadowski 2016 suggests employing new and emerging capabilities "is not like integrating a better rifle into the platoon... it has the potential to change the formation itself and the character of future warfare".

\section{POSSIBLE APPROACHES}

Other organisations have developed various approaches to address the challenge of developing and assessing future concepts and capabilities. Consideration of these approaches shows that there was no ready-made method that could be employed, but aspects of a number of approaches were relevant to the $2018 \mathrm{AC}$.

\subsection{Concept Development and Experimentation Frameworks}

Concept Development and Experimentation (CD\&E) is a widely used term for a methodology employed to inform transformational challenges in defence, such as the development of future military capability (Wiel et al, 2010). A well-known CD\&E framework is that developed by NATO and described in the NATO Concept Development and Experimentation Handbook 2018.

The NATO CD\&E Handbook 2018 describes a process of spiral development to create robust and rigorous concepts where analysis and experimentation are key enablers. This process provides a framework to 'iteratively explore, test, refine, and validate concepts' using 'progressive elaboration' knowledge gained to further refine requirements, solutions and approaches. To do this the NATO CD\&E process employs three separate types of experiments within the CD\&E process, these are listed below;

1. Discovery Experiments that introduce novel systems, concepts, organisational structures and technology and seek potential solutions and determine new information

2. Hypothesis Experiments that test to prove or disprove a hypothesis

3. Validation Experiments that confirm suitability of proposed solution to achieve desired outcomes or demonstrate evidence of ability to improve mission effectiveness

The NATO CD\&E approach provided a useful guide to how to approach the 2018 campaign, with the discovery experiment being the most relevant to the 2035 BG problem (with no intent to progress to concept approval and implementation in the campaign timeframe). However, there is a lack of detail on the concept development process; with the emphasis on analysis and experimentation to test and refine concepts, rather than developing them. 
Broadly speaking the NATO CD\&E spiral process of research, development and refinement/validation was applied in the DCDE approach; the key difference was the application of non-linear Design Thinking methods. For example, experimentation and analysis were conducted during the concept design phase bringing elements of refinement and testing (validation) into the development of draft concepts to support design of a future force option. This included use of Red Teaming and wargaming to progressively elaborate and rapidly iterate design, analysis and refinement. In addition, DCDE activities used all three types of experimentation in the one event, enabling exploration, testing and proposal of refinements whilst building evidence to inform decision makers. Further, subsequent activities were deliberately planned to be responsive and adaptable to the findings of previous activities to capitalise on opportunities for further discovery, testing and refinement.

The NATO CD\&E process was applied in the NATO Urbanisation 2035 project a five year concept exploration and development study focused on Urban Mega Cities envisaged for a possible 2035 future environment. The NATO Urbanisation 2035 project included concept writing workshops, scenario design workshops and a validation wargame (Bodnar and Collins 2019). The 2018 AC was similar to the concept exploration and development stages of the NATO Urbanisation 2035 project with a focus on close combat in urban environments. The key differences were the timeframe (12months for the $2018 \mathrm{AC}$ versus five years for the NATO Urbanisation 2035 project, and the development of a 2035 BG structure in the 2018 AC.

\subsection{US Army Campaign of Learning or Future Warfare Study Plan (Unified Quest Series)}

The US Army applies a two year campaign of learning through a series of events that challenge ideas, concepts and required capabilities to anticipate challenges and evaluate the viability of draft joint concepts (U.S. Army War College Archives 2013). The Unified Quest consisted of multiple events including future technology seminars, operational design seminars and seminar wargames to explore alternative concepts for current and future conflicts (Association of the United States Army 2006). Of particular interest is the Unified Quest Deep Future Wargame which investigated emerging tactical, operational and strategic challenges in a plausible future operational environment to inform operational-level concepts and investment options transitioning from an infusion of key technologies to exploring new unit formations (Future Warfare Division 2013).

The DCDE approach is similar to the US Army Campaign of Learning in that it applied a series of activities with rapid insights provided to inform both current and future decisions. However, the DCDE wasn't limited to seminar wargames and analysis but progressed to more detailed refinement including combat simulation using a multi-method approach focused on the design of an aspirational future option including concepts and capabilities.

\subsection{Disruptive Technology Assessment Game}

López-Vicente and Rademaker 2011 describes a Disruptive Technology Assessment Game (DTAG) as a tool for assessing the impact of concepts and technologies in a two sided tabletop game combining military knowledge and technical expertise. The DTAG involves (1) identification of relevant technologies, (2) creation of concepts, conduct of wargames (4) assessment of outputs, based on a comparison of technologyenhanced and baseline cases.

The approach appears useful as an element within a campaign to explore the relative benefits and military utility of different technology insertions, which could feed into subsequent simulation activities within a campaign construct. However, the 2018 AC was required to develop new concepts for the collective use of emerging technologies within new BG structures; not just examine technology insertion to existing structures and concepts and impact assessment as for the DTAG process.

\subsection{Systemic Design}

Systemic Design combines elements of Design Thinking and systems thinking that can be used to generate innovative solutions to complex, ill-structured problems (Ryan 2013). A key characteristic of Design Thinking is the interplay and iteration between divergence, to explore and understand the problem space, and convergence, to identify and test solutions. Pincombe et al, 2017 applied systemic design to the challenge of designing a future force in the 2050 timeframe. The systemic design method employed a range of techniques including current system mapping, foresighting, idealised design, and detailed design, including the development of paper-based prototypes, which allowed the viability of concepts for the future force to be tested. Whilst the approach did not develop a force structure at the level of detail required for the 2018 campaign, the value of the approach was clearly demonstrated. 


\section{DESIGN, CONCEPT DEVELOPMENT AND EXPLORATION APPROACH}

Leveraging the approaches, described in the previous section we conducted the $2018 \mathrm{AC}$ and developed the Design, Concept Development and Exploration (DCDE) approach. A key feature of DCDE is the use of multiple smaller activities rather than fewer large activities, which allowed an action learning and iterative approach. This is supported by Perkins 2017 who notes that "no single experiment, wargame or tabletop exercise can delve into all ... nuanced dimensions... key stakeholders must view [experimentation] as "voyages of discovery" to be undertaken as part of a multi-year campaign". Similarly Woods and Greenwood 2018 suggest that a campaign of iterative activities (including workshops, wargames, constructing and virtual simulations and live field events) is required to generate sufficient evidence to develop and assess an idea.

The 2018 AC employed a range of methods including scenario development and foresighting, systemic design, structured interviews, red teaming, seminar wargaming, human-in-the-loop and closed loop combat simulation. A key aspect of the campaign was the use of wargaming for concept exploration through its ability to immerse participants, consistent with Woods and Greenwood 2018 use of experimentation during concept development to explore novel concepts incorporating emerging technologies.

Birker et al 1998 suggests establishing a concept operations group consisting of technologists, experienced military operators and generalist defence analysts. Accordingly, across the $2018 \mathrm{AC}$, a core working group was formed between Land Warfare Lab, Army Headquarters and Land Capability Analysis Branch, DST Group to provide military operators and defence analysts to maintain continuity of knowledge. Technology SMEs were brought in from Academia, Industry and from other branches of DST Group as required to ensure use of technology in the 2035 BG was consistent with likely technology maturity in that timeframe.

Due to the 12 month timeframe, the $2018 \mathrm{AC}$ focused on one scenario (close combat) and one force option (2035 BG), anticipating that subsequent years would build on the first year's activities through testing the initial force option in different scenarios as well as exploring other future force options. A key stakeholder requirement was the need for the $2035 \mathrm{BG}$ to fight against a peer adversary, with the same set of capabilities for both sides. This provided concepts for the 2035 timeframe from both Blue (offensive) and Red (defensive) perspectives and encouraged both sides to adapt and challenge existing practices, as suggested by Ellis-Smith 2016.

\subsection{Campaign Activities}

Using the DCDE approach, the 2018 AC was conducted in four key phases; align, design, analyse and refine. The align phase was focused initially on understanding key drivers for 2035. This included a review of the $2035 \mathrm{OE}$ and strategic context to develop force design criteria for the 2035 timeframe. Alignment continued throughout the campaign in order to reflect sponsor and key stakeholder perspectives as their understanding of the problem evolved.

The design phase used a systemic design approach to explore future 2035 force options exploiting Robotics \& Autonomous Systems, and other emerging technologies. The initial activity, in which the force options generated were unconstrained by the IIP realised (funded) force and with optimistic interpretations of technology maturity in 2035, allowed for genuine innovative exploration and helped participants gain insights into future possibilities. Subsequent activities in the design phase combined the insights gained from the initial activity with a consideration of the constraints imposed by the funded force and a more pragmatic estimate of technology maturity for 2035. This resulted in a 2035 BG design that was based on insertion/addition of key technologies and systems to a $2028 \mathrm{BG}$ based on the funded force. The output from the design phase included both an improved understanding of key aspects of the $2035 \mathrm{OE}$ as well as a welldefined BG organisational structure underpinned by well-formed concepts and system descriptions, which were refined by a number of red-teaming activities that linked the design and analyse phases.

The analyse phase of the $2018 \mathrm{AC}$ included an analytical seminar-style wargame, human-in-the-loop combat simulation based wargaming and closed loop simulation to explore and assess novel concepts and key support requirements of the $2035 \mathrm{BG}$, in comparison to the $2028 \mathrm{BG}$. A key focus was on the reliance of the 2035 BG on support from Divisional / Joint Task Force assets and the sensitivity to key constraints that could influence the integration and coordination of effects deemed critical to the BG effectiveness. The refine phase ran iteratively throughout the $2018 \mathrm{AC}$ using adaptive red teaming, to inform updates to the $2035 \mathrm{BG}$ design. The refine phase also included consistency, thematic and trade-space analysis, conducted throughout the campaign, to build evidence for upcoming investment and modernisation decisions.

The final output from the 2018 campaign was an aggregation of the results from all individual activities and additional analysis and refinement including thematic analysis across activities, and analysis of simulation 
replays. The key outputs from this analysis was a set of concepts for the 2035 BG and insights into its performance.

\subsection{Overview of Findings}

Key aspects of the $2035 \mathrm{OE}$ for the 2018 AC included pervasive unmanned aerial systems and sensors, the lethality and availability of precision munitions, increased capabilities to counter fires, increased availability of electronic warfare effects, and advances in networking leading to highly collaborative effects. The 2035 BG is characterised by numerous Robotic and Autonomous Systems (RAS) enabled systems that provide enhanced ISR, EW and fires down to section level. Battle books describing the Blue and Red concepts and major systems were continually refined during the campaign and are summarised as follows:

- Capabilities and effects will be available at much lower tactical levels than they are currently

- Networking and agile C2 is required to enable orchestration and layering of effects to achieve both survivability and lethality effects at the lowest levels

- RAS-enabled systems increased situational awareness (SA) at combat team and below through increased availability of organic sensors (ground- and air-based)

- Pervasive surveillance causes the need for improved deception and decoy concepts

- EW effects particularly those available on RAS-enabled systems provide mechanisms for deception and decoy as well as to undermine counter-fires capabilities

- RAS-enabled systems increased the effectiveness of highly lethal and distributed fires

- Collective capabilities for Counter Rocket Artillery and Missiles (CRAM) and Counter Unmanned Air Systems (CUAS), were essential to survive the highly lethal and pervasive surveillance capabilities prominent in the $2035 \mathrm{OE}$

- Novel concepts are required to fully exploit the opportunities that multi-role RAS-enabled systems provide, across multiple warfighting functions

The advantages of the 2035 BG are predicated on the realisation of sufficient technological advances, particularly in autonomy, AI and networking; if this does not occur then these advantages may not be realised and current capabilities would need to be evolved by alternative approaches

\section{DISCUSSION}

\subsection{Utility of the Approach}

The DCDE approach took elements of all of the methods described in Section 2, but the key differences were; an emphasis on design which was incorporated into all campaign activities, and use of wargaming to immerse participants to enable exploration rather than for conducting assessment. The ability to iterate concept development was important and could be increased in future campaigns by allowing additional opportunities to revisit, and if necessary discard ideas. There was a continual tension between design and assessment with a desire for exploration balanced by the need for tangible outcomes from analysis and refinement. There were also difficulties in representing some novel systems and effects for wargaming and simulation and this is an area for further investigation.

The DCDE approach used in the 2018 AC provided ample information to inform the Land Force design, and highlight potential areas for further experimentation and investment. However, to have more impact, senior leaders will need to overcome organisational and culture barriers and repeated failure of military change management which Kidson 2017 describes as 'culture capture' and garner support for change to enable innovation and adaptation. This includes recognising Pink Flamingos ${ }^{1}$, known knowns that are ignored and become inevitable surprises to those trapped in rigid bureaucratic decision making structures (Hoffman 2015) and taking action. To mitigate this, the $2018 \mathrm{AC}$ used a partnered reporting process to brief key stakeholders before, during and after activities using existing committee processes. Ultimately the option developed and explored through the 2018 AC will have impact through resultant discussion and professional debate with ongoing input to subsequent future land force design activities.

\subsection{Comparison of Findings}

The findings from the $2018 \mathrm{AC}$ are similar to those of other studies and defence analyst discussion papers. The key addition to the $2018 \mathrm{AC}$ was contextualisation to the Australian $2035 \mathrm{OE}$ and the direct comparison

\footnotetext{
${ }^{1}$ Digital photography was a pink flamingo to Kodak
} 
of the 2035 BG with the current and funded force. The latter outcome demonstrated that there are significant advantages from RAS and AI capabilities and effects being resident within lower tactical levels. A similar potential future force design to the 2035 BG is the Multi-domain Dragoons Squad described by Becker 2018 The Dragoon Squad is a small unit that combines the all-terrain, close fight capabilities of infantry with the operational mobility and flexibility of mounted cavalry with organic indirect fires and robotic and autonomous ground and air systems based on the 2035-2050 timeframe. While the 2035 BG is based on a larger mechanised formation, the addition of capabilities and effects at the lowest possible level and novel concepts for employment of RAS are similar.

Key findings from the Unified Quest Deep Futures Wargame 2018 emphasise the importance of networks and the proliferation of AI-enabled systems to utilise multi-functional RAS that will be available at echelons below brigade. Additionally the U.S. Army War College (2018) suggest that employment of ubiquitous RASenabled vehicles will require changes to current tactics, techniques, and procedures to be successful in the future. Further Andresky and Henderson (2018) say that a new approach to command and control (C2) of RAS enabled formations will be required. Collectively, these findings support the 2018 AC finding that novel concepts will be required to fully realise the potential of emerging technology and fundamental changes may be possible to reduce the size of a formation while enhancing its effectiveness.

López-Vicente and Rademaker 2011 explore potential future technology insertions and as with the 2018 AC they assume that major platforms and systems remain in-service. Notably this is similar to the $2018 \mathrm{AC}$ as it is not only technology/platform insertion and that replacements may not be "like with like" but the concepts of use and employment that enhance the capability provided to the $2035 \mathrm{BG}$.

The NATO Capstone Concept for Joint Military Operations in an Urban Environment states that implementation of successful future concepts will require adaptive capabilities and adoption of new technologies, and an overall change in mindset (NATO 2018). This change in mindset is one of the challenges identified during the 2018 AC to allow concept designers the freedom to explore different and potentially culturally repugnant ideas to exploit the potential of emerging technologies. Furthermore, if these ideas provide significant advantages there is a need for a champion to argue for continued research, development and implementation.

\section{CONCLUSION}

The DCDE approach built upon findings from each activity and was sufficiently flexible to respond to changes in stakeholder priorities throughout the campaign. This enabled significant exploration as well as generating an evidence base to support modernisation decisions now and for a flexible and agile future force. The ability to encourage innovation and development of new and novel concepts was enhanced by immersion in context through use of seminar and combat simulation wargaming. Further, the use of a core working group across all activities was critical in maintaining continuity of knowledge.

The DCDE approach focused on both exploration and evaluation and as a result the 2035 BG that was developed incorporated a range of new and novel concepts and capabilities. Further investigation is necessary to examine other scenarios and to design alternative future force options, including considering other emerging technologies. This will be supported by further application and development of the DCDE approach.

\section{ACKNOWLEDGEMENTS}

The authors would like to acknowledge the contributions of Fred DJ Bowden and Nicholas Kempt to the design of the analysis phase and long term contribution to Concept Exploration approaches. The authors would also like to acknowledge the support provided through partnership with the Land Warfare Laboratory, particularly that of LTCOL David Klomp and LTCOL (USMC) James Nolan.

\section{REFERENCES}

Association of the United States Army (2006) The Unified Quest Campaign of Learning: Preparing the Force for Future Operations, Torchbearer Special Report April 2006

Australian Government (2017), 2017 Foreign Policy White Paper, p. iii

Becker, J. (2018) How Lethal, Mobile, Protected and Aware? Exploring the Art of the Possible in Future Infantry Combat, Small Wars Journal https://smallwarsjournal.com/jrnl/art/how-lethal-mobile-protected-andaware accessed 04 Jun 19 
Birker J, Neu C R, Kent G "Gaining New Military Capability: An Experiment in Concept Development" RAND 1998

Bodnar J and Collins S "NATO Joint Military Operations in an Urban Environment: A Capstone Concept", The Three Swords Magazine 34/2019 pp 93-96

Campbell, A (2017) Transcript of the speech presented to the Royal United Services Institute (United Kingdom), Whitehall, London on Friday 8 December 2017, Australian Army Journal Autumn 2018, Volume XIV, No 1

Ellis-Smith, J (2016), 'Challenging our approach to conventional war: another view on Ukraine', Land Power Forum [blog], 18 August 2016, http://www.army.gov.au/Our-future/Blog/Articles/2016/08/Lets-learntheright-lessons-from-Ukraine accessed 29 November 2016.

Future Warfare Division (2013), Unified Quest 2013 Deep Future Wargame 2030-2040 Executive Report, Army Training and Doctrine Command, Army Capabilities Integration Center, Future Warfare Division, Sep 2013

Greenwood T and Greer J (2018) "Experimentation: The Road to Discovery", https://thestrategybridge.org/the-bridge/2018/3/1/experimentation-the-road-to-discovery accessed 15 May 19

Hoffman, F. (2015) Black Swans and Pink Flamingos: Five Principles for Force Design, https://warontherocks.com/2015/08/black-swans-and-pink-flamingos-five-principles-for-force-design/ accessed 21 May 19

Kidson, R. (2017) Force Design in the 1990s: Lessons for Contemporary Military Change Management, $A n$ Australian Army History Unit Monograph, Commonwealth of Australia

López-Vicente and P. Rademaker, M (2011), Disruptive Technology Assessment Game, NATO Research and Technology Information Systems Technology Panel Symposium 7 - 8 May 2011

NATO (2018) Concept Development and Experimentation Handbook, Allied Command Transformation, NATO 2018

NATO (2018) Capstone Concept - Joint Military Operations in an Urban Environment, Allied Command Europe and Transformation NATO November 2018

Perkins, D. (2017), Multi-Domain Battle: The Advent of 21st Century, War. Military Review. Nov-Dec 2017

Ryan, A. (2013). A Theory of Systemic Design" in: B. Sevaldson \& P. Jones (Eds.) Relating Systems Thinking and Design 2013 Symposium Proceedings https://systemic-design.net/wpcontent/uploads/2013/12/Ryan.pdf

Pincombe, B. Ryan, A. Kempt, N. Stephens, A. Tomecko, N. Reid, D. \& Tang, K. (2017). Systemic Design of a Force for the Australian Army in 2050. Accepted for publication in the ADF Journal special issue.

Sadowski, R.W. (2016) Enabling MUM-T within Army Formations, U.S. Army Tank Automotive Research Development and Engineering Centre, Robotics Community of Practice presentation 30 Jan 2016

Schmitt, J.F. (2002), A Practical Guide for Developing and Writing Military Concepts, Defense Adaptive Red Team Working Paper \#02-4, McLean, VA: Hicks \& Associates, December 2002

Smith, C. (2018), On Future Thinking and Innovation: How Military Concept Writing can unwittingly suppress Innovation, Australian Army Journal Autumn 2018, Volume XIV, No 1

U.S. Army War College Archives (2013), Unified Quest: Preparing for America's Next First Battle https://www.armywarcollege.edu/news/Archives/12061.pdf accessed 15 May 18

U.S. Army War College, (2018) Unified Quest 2018 Deep Future Wargame Summary Report http://arcicsem.azurewebsites.us/App_Documents/UQ/Unified-Quest-18-Deep-Future-Wargame-Summary-

Report FINAL.PDF accessed 04 Jun 19

Wiel, W. Weime, I. Hasberg, M. \& Husikamp, W. (2010). Concept Maturity Levels: Bringing Structure to the CD\&E Process. Proceedings of the 2010 Interservice/Industry Training, Simulation, and Education Conference.

Woods K and Greenwood T (2018), Multi-Domain Battle: Time for a Campaign of Joint Experimentation, Joint Forces Quarterly Jan 2018. 